\title{
Phase retrieval from a high-numerical-aperture intensity distribution by use of an aperture-array filter
}

\author{
Nobuharu Nakajima \\ Faculty of Engineering, Shizuoka University, 3-5-1 Johoku, Naka-ku, Hamamatsu, \\ 432-8561, Japan
}

Abstract

Almost all non-interferometric phase retrieval methods used in coherent diffractive imaging have been based on the measurement system with low numerical aperture, in which Fresnel or Fraunhofer approximation is valid to express the wave propagation between an object and a detector. In microscopy, which is a typical application of coherent diffractive imaging, the measurement of the diffraction intensity with high numerical aperture is required for the object reconstruction at high spatial resolution. We here propose an extension procedure to apply the previous phase retrieval method using an aperture-array filter [J. opt. Soc. Am. A 25, 742] to the system with high numerical aperture, in which the first Rayleigh-Sommerfeld integral for spherical waves is utilized instead of the Fresnel integral for parabolic waves. Computer-simulated examples in the high-numerical-aperture system demonstrate the object reconstruction at high lateral resolution and the retrieval of the information in depth direction of an object.

OCIS codes: 100.0100 Image processing, 100.5070 Phase retrieval 


\section{INTRODUCTION}

Lensless coherent imaging from the diffraction intensity of an object, in principle, provides wavelength limited resolution without any deleterious effect of the aberration and the finite extent of a lens. Such an imaging approach, however, requires that the phase of the diffraction field is retrieved by some method. Many approaches for the diffractive imaging have been proposed, for example, in-line [1-3] or off-axis [4] holography, holographic imaging with extended reference containing a sharp corner [5,6] or a uniformly redundant array [7], noninterferometric imaging by iterative [8-10] or non-iterative [11,12] phase retrieval methods, and so forth. Recently, coherent x-ray diffractive imaging by the non-interferometric methods has attracted considerable attention [13]. In imaging systems for these methods, a reference wave is not required and the illumination beam needs to be spatially coherent only over the extent of the object, so that the implementation of the systems is substantially simpler than holography. For example, object reconstruction by use of the iterative method has been demonstrated in x-ray experiments for artificial [14-16] and biological [17] objects. In addition, the transport-ofintensity equation method, which is one of non-iterative methods, has been applied to x-ray imaging for some materials $[18,19]$.

A typical application of coherent diffractive imaging is microscopy, in which high spatial resolution is usually pursued. Therefore the intensity in the diffraction plane is required to be recorded with high numerical aperture (NA). Currently used non-interferometric phase retrieval methods, however, have their limitations when they are applied to high-NA intensity data. Until now, almost all non-interferometric phase-retrieval methods have required the Fresnel or Fraunhofer approximation for the diffraction calculation. For example, the Fourier transform relationship appeared in the Fresnel or Fraunhofer diffraction integral is essential for the iterative 
phase-retrieval methods, because such iterative methods are based on the algorithm of bouncing back and forth between the object domain and the Fourier domain. The noniterative phaseretrieval methods based on the transport of intensity equation also require the paraxial wave propagation for the solution of the intensity equation.

We have recently proposed $[20,21]$ a deterministic phase retrieval method using an aperture-array filter to reconstruct a complex-valued object from a single diffraction intensity pattern, of which the theoretical development is also based on the Fresnel integral. In this paper, an extension procedure of the previous method is proposed to apply this method to the system with high NA, in which the first Rayleigh-Sommerfeld integral for spherical waves is utilized for the diffraction formula instead of the Fresnel integral for parabolic waves. To the best of my knowledge, this is the first non-interferometric (i.e., non-holograhpic) phase-retrieval method from a high-NA intensity distribution beyond the region of Fresnel diffraction.

In Section 2, we consider the relation between the limitation of the Fresnel approximation and the NA of the measurement system in coherent diffractive imaging, and develop an improved procedure for the object reconstruction from high-NA diffraction intensity by the phase retrieval method using an aperture-array filter on the basis of the first RayleighSommerfeld integral. In Section 3, the validity of the present method is demonstrated with computer simulations of the object reconstruction at high-resolution and the retrieval of the object's depth information from a high-NA intensity distribution. Concluding remarks are given in Section 4. 


\section{Formulation of Object reconstruction}

\section{A. Limitation of the Fresnel approximation}

In the application of coherent diffractive imaging to microscopy, the intensity in the diffraction plane is required to be recorded with high NA for the sake of high spatial resolution. Until now, almost all of the coherent diffractive imaging by non-interferometric phase retrieval methods have utilized low-NA measurement systems under the Fresnel or Fraunhofer approximation. Thus we consider here the relation between the limitation of the Fresnel approximation and the NA of the measurement system.

We assume that the object plane and the diffraction plane are parallel and denoted by the coordinates $(u, v)$ and $(x, y)$, respectively. The distance between the two planes is designated by $z$. The Fresnel approximation is valid when the object extent and the extent of the diffraction plane are smaller than the distance $z$ [22]:

$$
z^{3}>\frac{\pi}{4 \lambda}\left[(x-u)^{2}+(y-v)^{2}\right]_{\max }^{2}
$$

where $\lambda$ is the wavelength of illuminating light. In applications to microscopy, the extent of the diffraction plane can be assumed to be larger than that of the object ( $u \square x, v \square y$ ). For simplicity, we here restrict to considering the condition along the $x$ axis of the diffraction plane with a square extent of side length $H$. Then the distance $z$ at the limitation of the Fresnel approximation is given by

$$
z=\left[\pi(H / 2)^{4} / 4 \lambda\right]^{1 / 3}
$$

The NA of the measurement system in the direction of $x$ axis is defined by 


$$
\mathrm{NA}=\sin \theta=\frac{H / 2}{\left[z^{2}+(H / 2)^{2}\right]^{1 / 2}},
$$

where $\theta$ is the angle between the outward normal vector in the object plane and the vector pointing from the origin in the object plane to a point $(H, 0)$ in the diffraction plane.

Then the lateral resolution $\delta_{l}$ in the object plane can be defined [23] by

$$
\delta_{l}=\frac{\lambda}{2 \mathrm{NA}}
$$

Eliminating $H$ from Eqs. (2) and (3), we obtain the NA at the limitation of the Fresnel approximation:

$$
\mathrm{NA}_{\text {limit }}=\left(\frac{2 \sqrt{\lambda}}{\sqrt{\pi \mathrm{z}}+2 \sqrt{\lambda}}\right)^{1 / 2} .
$$

For example, if the observation distance $z$ is $10^{4}$ times the length of $\lambda$, the $\mathrm{NA}_{\text {limit }}$ becomes about 0.106 . Therefore, the rigorous treatment for the diffraction is necessary for the object reconstruction with higher resolution than $\delta_{l}=\lambda / 2 \mathrm{NA}_{\text {limit }}$.

\section{B. Reconstruction from a high-NA intensity distribution}

We here present the extension of the non-iterative (deterministic) phase retrieval method using an aperture-array filter to the object reconstruction from a diffraction intensity recorded at high numerical aperture beyond the $\mathrm{NA}_{\text {limit }}$ in Eq. (5). Figure 1 shows a schematic diagram of the 
measurement system. This layout consists of three planes (object, diffraction, and detector), which are perpendicular to an optical axis. The two distances of the object-to-diffraction planes and the diffraction-to-detector planes are designated by $z$ and $l$, respectively. We assume that an object is illuminated by a coherent plane wave with a wavelength $\lambda$. The object plane is defined as the plane immediately behind the object. Under the scalar theory of diffraction, we here refer to the complex amplitude distribution $f(u, v)$ (which is assumed to be of finite extent $\sigma$ ) in the object plane as the object function to be reconstructed. The complex amplitude distribution $F(x, y)$ in the diffraction plane is given by the first Rayleigh-Sommerfeld integral [22]:

$$
F(x, y)=\frac{1}{2 \pi} \iint_{\sigma} f(u, v) \frac{\exp (i k r)}{r}\left(\frac{1}{r}-i k\right) \frac{z}{r} d u d v
$$

where $k$ is the wave number, and

$$
r=\left[z^{2}+(x-u)^{2}+(y-v)^{2}\right]^{1 / 2} .
$$

According to the convolution theorem, we can rewritten as

$$
F(x, y)=\mathfrak{J}^{-1}\left\{\mathfrak{I}[f(u, v)] \times \exp \left[i \frac{2 \pi z}{\lambda} \sqrt{1-(\lambda \mu)^{2}-(\lambda v)^{2}}\right]\right\},
$$

where $\mathfrak{I}[\cdots]$ and $\mathfrak{I}^{-1}[\cdots]$ denote the Fourier and the inverse Fourier transforms, respectively, and the following explicit form was used for the Fourier transform of the diffraction kernel [24]:

$$
\mathfrak{J}\left[\frac{1}{2 \pi} \frac{\exp (i k r)}{r}\left(\frac{1}{r}-i k\right) \frac{z}{r}\right]=\exp \left[i \frac{2 \pi z}{\lambda} \sqrt{1-(\lambda \mu)^{2}-(\lambda v)^{2}}\right],
$$

in which $\mu$ and $v$ denote the spatial frequencies. Consequently, the object function can be reconstructed by deconvolving the complex amplitude distribution $F(x, y)$ consisting of the 
measured modulus $|F(x, y)|$ and the retrieved phase $\phi(x, y)$ (i.e., $F(x, y)=|F(x, y)| \exp [i \phi(x, y)])$

Thus, to retrieve the phase, an array filter, consisting of $N \times M$ square apertures each of width $w$ distributed over a Cartesian grid of period $d$, is inserted in the diffraction plane to take a correlation of the square function and the field distribution of the diffracted wave from the object. We assume that the parameters $d, N$, and $M$ fulfill the sampling condition for encoding the intensity distribution of the diffracted wave in the diffraction plane. As we shall see, if the diffraction pattern of each aperture in the detector plane is isolated approximately from those of the adjoining apertures, one complex amplitude for each aperture in the diffraction plane can be recovered from the data of the diffraction intensities from all of the apertures in the detector plane by regarding the data as the Fresnel diffraction intensities from a blurred object function, and then a high-resolved object function is reconstructed by back-propagating the retrieved complex amplitude distribution with the exact diffraction formula. Thus we calculate the intensity distribution in the detector plane under the above conditions.

Using the first Rayleigh-Sommerfeld integral again, the complex amplitude $K(\xi, \eta)$ in the detector plane is given by

$$
K(\xi, \eta)=\frac{1}{2 \pi} \sum_{n=-N / 2}^{N / 2-1} \sum_{m=-M / 2}^{M / 2-1} \iint_{-\infty}^{\infty} F(x, y) R\left(x-x_{n}, y-y_{m}\right) \frac{\exp \left(i k r^{\prime}\right)}{r^{\prime}}\left(\frac{1}{r^{\prime}}-i k\right) \frac{l}{r^{\prime}} d x d y
$$

where

$$
r^{\prime}=\left[l^{2}+(\xi-x)^{2}+(\eta-y)^{2}\right]^{1 / 2}
$$

and $R\left(x-x_{n}, y-y_{n}\right)$ denotes the amplitude transmittance of a square aperture being at the position of the coordinates $\left(x_{n}, y_{m}\right)=(n d, m d),[R(x, y)=1.0$ for $-w / 2 \leq x \leq w / 2$ and 
$-w / 2 \leq y \leq w / 2$, and $R(x, y)=0$ otherwise]. In applications to microscopy, we may assume that the variation of the wave front incident on each square aperture is gradual and so its diffraction angles from each aperture are small, because the extent of the object is very small compared with the distance $z$ between the object and the diffraction planes. Therefore the complex amplitude diffracted from each aperture can be calculated using the Fresnel approximation:

$$
K(\xi, \eta)=\frac{\mathrm{e}^{i k l}}{i \lambda l} \sum_{n=-N / 2}^{N / 2-1} \sum_{m=-M / 2}^{M / 2-1} \iint_{-\infty}^{\infty} F(x, y) R\left(x-x_{n}, y-y_{m}\right) \exp \left\{i \frac{\pi}{\lambda l}\left[(\xi-x)^{2}+(\eta-y)^{2}\right]\right\} d x d y .
$$

Note that the simulated wave propagation in Sec. 3 is calculated not using Eq. (10) but using Eq. (9) to validate the present method.

We suppose that the discrete intensities at the points of coordinates $\xi_{n}=x_{n}(1+l / z)$ and $\eta_{m}=y_{m}(1+l / z) \quad(n=-N / 2 \cdots, 0, \cdots N / 2-1, \quad$ and, $\quad m=-M / 2 \cdots, 0, \cdots M / 2-1) \quad$ in the detector plane are observed according to the procedure for phase retrieval in the previous paper [21], where the factor $(1+l / z)$ indicates the extension of the diffraction pattern of the array in the detector plane due to the effect of wave propagation from the object. Substituting the coordinates $\xi_{n}=x_{n}(1+l / z)$ and $\eta_{m}=y_{m}(1+l / z)$ into Eq. (10), we obtain

$$
\begin{aligned}
K\left[x_{n}(1+l / z), y_{m}(1+l / z)\right] & =\frac{\mathrm{e}^{i k l}}{i \lambda l} \sum_{n^{\prime}=-N / 2}^{N / 2-1} \sum_{m^{\prime}=-M / 2}^{M / 2-1} \rho \iint_{-\infty}^{\infty} \exp \left[-i \frac{\pi}{\lambda z}\left(x^{2}+y^{2}\right)\right] F(x, y) \\
\times & R\left(x-x_{n^{\prime}}, y-y_{m^{\prime}}\right) \exp \left\{i \frac{\pi}{\lambda l}\left(1+\frac{l}{z}\right)\left[\left(x-x_{n}\right)^{2}+\left(y-y_{m}\right)^{2}\right]\right\} d x d y,
\end{aligned}
$$

where $\rho=\exp \left[i \pi(1+l / z)\left(x_{n}{ }^{2}+y_{m}{ }^{2}\right) / \lambda z\right]$. We assume that the central part of the diffraction pattern of each aperture is hardly affected by the diffraction patterns of the adjoining apertures. Then the observable intensity of Eq. (11) may be represented by 


$$
\left|K\left[x_{n}(1+l / z), y_{m}(1+l / z)\right]\right|^{2} \cong\left|\iint_{-\infty}^{\infty} \exp \left[-i \frac{\pi}{\lambda z}\left(x^{2}+y^{2}\right)\right] F(x, y) R^{\prime}\left(x-x_{n}, y-y_{m}\right) d x d y\right|^{2},
$$

where $R^{\prime}(x, y)$ is the aperture function including the quadratic phase:

$$
R^{\prime}(x, y)=R(x, y) \exp \left[i \frac{\pi}{\lambda l}\left(1+\frac{l}{z}\right)\left(x^{2}+y^{2}\right)\right]
$$

and unimportant multiplicative constant $1 / \lambda l$ is omitted.

A sufficient condition for the isolation of the central parts of the diffraction patterns from the apertures would be that the main width of the diffraction pattern of each aperture is smaller than the period of the array pattern projected on the detector plane. Since the range of the diffraction angle from each aperture are small as described above, the condition derived in the previous paper [21] can be also used in the present case. The main width of the diffraction pattern from each aperture in the detector plane was defined by $\varepsilon(2 \lambda l / w+l \sigma / z)$, where $2 \lambda l / w$ denotes the main lobe between the first two zeros of the sinc function (i.e., the Fourier transform of the square aperture), $l \sigma / z$ is the extent predicted by geometrical optics for the object with finite extent $\sigma$, and $\varepsilon$ is set to be a constant larger than unity in compensation for the extension due to the Fresnel diffraction. This main width can be roughly estimated from the calculated diffraction pattern of the aperture function $R^{\prime}(x, y)$ and the rough extent of the object, which is obtained by another measurement. On the other hand, the period of the projection pattern of the array in the detector plane is given from the geometrical optics prediction as $d(1+l / z)$, because the object's extent in applications to microscopy is very small as compared with the extent of the aperture-array filter. Consequently, if the condition $\varepsilon(2 \lambda l / w+l \sigma / z)<d(1+l / z)$ is satisfied in the measurement system, the summation sign in Eq. (11) can be omitted and so Eq. (12) may be 
regarded as the intensity at the coordinates $\xi=x_{n}(1+l / z)$ and $\eta=y_{m}(1+l / z)$ in the detector plane.

In the previous paper [21], we have presented the method of the phase retrieval from periodically sampled intensities at coordinates $\left(\xi_{n}, \eta_{m}\right),\left(\xi_{n} \pm \tau, \eta_{m}\right)$, and $\left(\xi_{n}, \eta_{m} \pm \tau\right)$ in the detector plane (where $\tau$ is a known constant). The previous method, however, is based on the Fresnel diffraction for the wave propagation between the object and the diffraction planes. An improved procedure is presented here, which extends the adaptable condition to the region required to be treated by the exact diffraction formula in Eq. (6). Thus we first investigate the information about the object, which is included in the correlation integral in Eq. (12), by use of Fourier transforms. From the properties of the Fourier transform, the inverse Fourier transform of the correlation integral in Eq. (12) can be written as

$$
\begin{aligned}
\mathfrak{I}^{-1}\left\{\iint_{-\infty}^{\infty} \exp \left[-i \frac{\pi}{\lambda z}\left(x^{2}+y^{2}\right)\right] F(x, y) R^{\prime}\left(x-x_{n}, y-y_{m}\right) d x d y\right\} \\
=\mathfrak{I}^{-1}\left\{\exp \left[-i \frac{\pi}{\lambda z}\left(x^{2}+y^{2}\right)\right] F(x, y)\right\} \times \mathfrak{I}^{-1}\left[R^{\prime}(x, y)\right],
\end{aligned}
$$

where the symmetry of the aperture function in Eq. (13) was used.

The first term of the transforms on the right-hand side of Eq. (14) is given by

$$
\begin{aligned}
\mathfrak{I}^{-1}\left\{\exp \left[-i \frac{\pi}{\lambda z}\left(x^{2}+y^{2}\right)\right] F(x, y)\right\}= & \iint_{-\infty}^{\infty} \exp \left[-i \frac{\pi}{\lambda z}\left(x^{2}+y^{2}\right)\right] F(x, y) \exp \left[i \frac{2 \pi}{\lambda z}(x u+y v)\right] d x d y \\
= & \exp \left[i \frac{\pi}{\lambda z}\left(u^{2}+v^{2}\right)\right] \\
& \times \iint_{-\infty}^{\infty} F(x, y) \exp \left\{-i \frac{\pi}{\lambda z}\left[(u-x)^{2}+(v-y)^{2}\right]\right\} d x d y .
\end{aligned}
$$


The convolution integral in Eq. (15) indicates that the exact complex amplitude distribution $F(x, y)$ in Eq. (6) is back-propagated by using the quadratic-phase exponential kernel valid for the Fresnel approximation. Therefore, if the inverse Fourier transform in Eq. (15) is calculated numerically, we obtain not the object function $f(u, v)$ but a blurred object function $f_{\mathrm{b}}(u, v)$ :

$$
\mathfrak{I}^{-1}\left\{\exp \left[-i \frac{\pi}{\lambda z}\left(x^{2}+y^{2}\right)\right] F(x, y)\right\}=\exp \left[i \frac{\pi}{\lambda z}\left(u^{2}+v^{2}\right)\right] f_{\mathrm{b}}(u, v) .
$$

Equation (16) implies that the inverse Fourier transform of the product of the exact diffraction amplitude distribution $F(x, y)$ and the quadratic phase $\exp \left[-i \pi\left(x^{2}+y^{2}\right) / \lambda z\right]$ corresponds to the product of the blurred object function $f_{\mathrm{b}}(u, v)$ and the quadratic phase $\exp \left[i \pi\left(u^{2}+v^{2}\right) / \lambda z\right]$. Thus, to retrieve the phase of the convolution integral in Eq. (12), we apply the procedure of phase retrieval in the previous paper to this case by regarding the exact diffraction amplitude distribution $F(x, y)$ as the Fresnel diffraction field from the blurred object function $f_{\mathrm{b}}(u, v)$. Substitution of Eq. (16) into Eq. (14) yields

$$
\begin{aligned}
\mathfrak{J}^{-1}\left\{\iint_{-\infty}^{\infty} \exp \left[-i \frac{\pi}{\lambda z}\left(x^{2}+y^{2}\right)\right] F(x, y) R^{\prime}\left(x-x_{n}, y-y_{m}\right) d x d y\right\} \\
=\exp \left[i \frac{\pi}{\lambda z}\left(u^{2}+v^{2}\right)\right] f_{\mathrm{b}}(u, v) r^{\prime}(u, v),
\end{aligned}
$$

where $r^{\prime}(u, v)$ denotes the inverse Fourier transform of the aperture function $R^{\prime}(x, y)$ in Eq. (13). To utilize the properties of entire functions for phase retrieval [25], we assumed in the previous paper [21] that the function $r^{\prime}(u, v)$ has an extent large enough to be approximated into a Gaussian function within the extent of the object function. It was shown in the previous paper [25] that the function $r^{\prime}(u, v)$ can be approximated into a Gaussian function with a quadratic phase, provided that a Fresnel number concerning the quadratic phase within the aperture width 
$w$ in Eq. (13) is less than 1.0. This approximation can be also applied to the present case, because the blurred object function $f_{\mathrm{b}}(u, v)$ has almost the same extent as the object $f(u, v)$, that is, the difference between $f_{\mathrm{b}}(u, v)$ and $f(u, v)$ is in high spatial frequencies due to replace the diffraction kernel for spherical waves by the quadratic-phase exponential. Therefore it is found that the periodically sampled intensities at coordinates $\left(\xi_{n}, \eta_{m}\right)$ in Eq. (12) contain the effect of the Gaussian filtering on the function $f_{\mathrm{b}}(u, v)$.

Using the same way as in Eqs. (10) to (12), we can obtain the intensity distribution at another points of coordinate $\left(\xi_{n} \pm \tau, \eta_{m}\right)$ in the detector plane:

$$
\begin{aligned}
\left|K\left(\xi_{n} \pm \tau, \eta_{m}\right)\right|^{2} & =\left|K\left[x_{n}(1+l / z) \pm \tau, y_{m}(1+l / z)\right]\right|^{2} \\
& \cong\left|\iint_{-\infty}^{\infty} \exp \left[-i \frac{\pi}{\lambda z}\left(x^{2}+y^{2}\right)\right] F(x, y) R^{\prime}\left(x-x_{n}, y-y_{m}\right) \exp \left(\mp i \frac{2 \pi x \tau}{\lambda l}\right) d x d y\right|^{2},
\end{aligned}
$$

where constant phase terms outside the integrals were omitted. Then the inverse Fourier transform of the correlation integral in Eq. (18) is given by

$$
\begin{aligned}
& \mathfrak{J}^{-1}\left\{\iint_{-\infty}^{\infty} \exp \left[-i \frac{\pi}{\lambda z}\left(x^{2}+y^{2}\right)\right] F\right.\left.(x, y) R^{\prime}\left(x-x_{n}, y-y_{m}\right) \exp \left(\mp i \frac{2 \pi x \tau}{\lambda l}\right) d x d y\right\} \\
&=\exp \left\{i \frac{\pi}{\lambda z}\left[(u \mp z \tau / l)^{2}+v^{2}\right]\right\} f_{\mathrm{b}}(u \mp z \tau / l, v) r^{\prime}(u, v) .
\end{aligned}
$$

This equation indicates that the correlation integral in Eq. (18) includes the influence of the Gaussian filtering on a displaced function $f_{\mathrm{b}}(u \mp z \tau / l, v)$ in the $u$ direction. This shift gives the information of the phase along the $x$ axis of the correlation integral in Eq. (12) to the intensity distribution in Eq. (18). In the same way, the sampled intensities at coordinates $\left(\xi_{n}, \eta_{m} \pm \tau\right)$ in the detector plane contain the information of the phase in the $y$ direction of the correlation 
integral in Eq. (12). Thus the two-dimensional phase of the correlation integral in Eq. (12) can be retrieved from those sampled intensities at coordinates $\left(\xi_{n}, \eta_{m}\right),\left(\xi_{n} \pm \tau, \eta_{m}\right)$, and $\left(\xi_{n}, \eta_{m} \pm \tau\right)$ in the detector plane by using the same phase calculation as in the previous paper [21]. In addition, the accuracy of the phase calculation can be improved by using multiple groups of sampled intensities for several values of $\tau$. The explanation of the calculation is omitted here since its specific details have been presented in the previous paper.

From the retrieved phase and the measured modulus, we can reconstruct an estimate of the correlation integral of $\exp \left[-i \pi\left(x^{2}+y^{2}\right) / \lambda z\right] F(x, y)$ and $R^{\prime}(x, y)$ in Eq. (12). The inverse Fourier transform of the estimated correlation integral yields the product of the blurred object function with the quadratic phase and the filter function as shown in Eq. (17). Dividing this product functions by the known filter function $r^{\prime}(u, v)$, and then Fourier transforming the resultant function, we obtain an estimate of $\exp \left[-i \pi\left(x^{2}+y^{2}\right) / \lambda z\right] F(x, y)$. Then an estimate of $F(x, y)$ can be calculated by compensating for the known quadratic phase $\exp \left[-i \pi\left(x^{2}+y^{2}\right) / \lambda z\right]$. Finally, a high-resolved object function $f(u, v)$ can be reconstructed by deconvolving the diffraction kernel for spherical waves from the estimate function $F(x, y)$ based on the double Fourier transform relationship in Eq. (7).

\section{COMPUTER SIMULATIONS}

The performance of the present method is demonstrated by computer simulation for the high-NA intensity measurement system. We assumed that an object was illuminated by a monochromatic 
soft-x-ray with wavelength $20 \mathrm{~nm}$. Data processing for calculating the first RayleighSommerfeld integral by means of a fast Fourier transform on the basis of the convolution theorem was carried out with $4096 \times 4096$ sampling points, of which the central part $(32 \times 32$ points) is the location of an object function and the sampling points except for the object function was padded with zeros. Note that such a large amount of zero padding is necessary for the calculation of the exact diffraction integral in the high-NA measurement. The distances between the object and the aperture-array and between the aperture-array and the detector in Fig. 1 were set to $z=0.416 \mathrm{~mm}$ and $l=0.0229 \mathrm{~mm}$, respectively. The aperture array was assumed to be uniform square grid of interval $d=2.85 \mu \mathrm{m}$ with $64 \times 64$ square apertures each of width $w=0.889 \mu \mathrm{m}$. The interval $d$ was determined using the sampling theorem for the rough extent of the object function, which is based on the Fourier transform relationship [as shown in Eq. (16)] between the complex amplitude distribution in front of the aperture-array filter and the blurred object function having almost the same extent as the object function. The NA and the $\mathrm{NA}_{\text {limit }}$ in Eqs. (3) and (5) for the present system become to be about 0.214 and 0.0881 , respectively. The parameters $z, l, d$, and $w$ were chosen to fulfills the requirement of the Gaussian approximation for the aperture function and the condition for the isolation of the central parts of the diffraction patterns from the apertures as described in Subsection 2.B. Note that we had to set such small values for the parameters $z, l, d$, and $w$ because of the limitation of the computer memory, but, in practical cases, these values can be increased with maintaining the same NA as in this case.

Figures 2 and 3 show the result of the first simulation. Figure 2(a) shows the modulus of the original object function consisting of seven Gaussian functions with no phase variation, where the physical size of the figure is assigned to be $1.42 \mu \mathrm{m} \times 1.42 \mu \mathrm{m}$, and is represented by $32 \times 32$ 
points. Figure 2(b) [or 2(d)] shows the modulus of the reconstructed object from noiseless (or noisy) modulus data of the measured intensity distribution at the coordinates $\left(\xi_{n}, \eta_{m}\right)$, $\left(\xi_{n} \pm \tau, \eta_{m}\right) \quad, \quad$ and $\quad\left(\xi_{n}, \eta_{m} \pm \tau\right) \quad\left[\quad \xi_{n}=n d(1+l / z) \quad\right.$ and $\quad \eta_{m}=m d(1+l / z) \quad$, $(n=-64 \cdots, 0, \cdots 63, m=-64 \cdots, 0, \cdots 63)]$ in the detector plane, where five values of $\tau$ at $0.0445 \mu \mathrm{m}$ spacing in a range of $0<\tau<0.180 \mu \mathrm{m}$ were utilized to improve the accuracy of the phase calculation according to the procedure in the previous paper [21]. In Fig. 2(c), the noiseless modulus data in the detector plane is shown on a base-10 logarithmic grey scale of a normalized modulus truncated to $10^{-4}$ for display purposes. For simulating the noisy intensities, normal random noises were produced by a computer and were added to the modulus data in the detector plane. When a factor of the signal-to-noise ratio (SNR) is defined by the ratio of the total intensities for the amplitudes and the noises in the detector plane, the SNR in Fig. 2(d) becomes 125. Figure 2(e) shows a blurred object function that is obtained by back-propagating the same retrieved amplitude distribution in the diffraction plane as used in Fig. 2(b) with the quadratic-phase exponential kernel valid for the Fresnel approximation as shown in Eqs. (15) and (16). The diffraction-limited lateral resolutions $\delta_{l}$ in Eq. (4) for $\mathrm{NA}=0.214$ and 0.0746 become to be about $0.0467 \mu \mathrm{m}$ and $0.134 \mu \mathrm{m}$, respectively. Therefore it is found that the lateral resolution in Fig. 2(b) is enhanced by a factor of about 2.87 for Fig. 2(e).

To evaluate the difference between an original and a reconstructed object, a measure of the quality of reconstruction is defined by the normalized root-mean-square (NRMS) error,

$$
\mathrm{ER}=\left[\frac{\sum_{u, v \in \sigma}\left|f(u, v)-f_{r}(u, v)\right|^{2}}{\sum_{u, v \in \sigma}|f(u, v)|^{2}}\right]^{1 / 2}
$$


where $f(u, v)$ and $f_{r}(u, v)$ are the original and the reconstructed object functions, respectively, and $\sigma$ denotes the extent of $f(u, v)$. Then the NRMS errors for the reconstructed objects in Figs. 2(b), 2(d) and 2(e) are 0.354, 0.373 and 0.598, respectively. From a comparison of Figs. 2(b), 2(d) and 2(e), it is seen that the results in Figs. 2(b) and 2(d) have been back-propagated correctly from the complex amplitude distribution including the information in the diffraction area beyond the Fresnel approximation.

Even if one value of the parameters $z, l, d$, and $w$ is changed to some degree from the correct value used in the calculation of the diffraction pattern, the reconstruction using the present method becomes not unstable but gradually worse. From computer simulations, it was found that the error of a few percent of the correct value for each parameter is within tolerance for the reconstruction with the NRMS errors below 0.5 in noiseless cases.

Next we investigated the NA valid for the Fresnel approximation in this measurement system. Figure 3 shows the results obtained from the retrieved complex amplitude distribution in the diffraction plane when varying NAs. The object reconstruction for the variation of the NA was simulated by back-propagating the product of the retrieved complex amplitude distribution in the diffraction plane and a stop-down filter to pass only the low spatial-frequency amplitude. Figures 3(a), 3(c) and 3(e) [or 3(b), 3(d) and 3(f)] are the results for NA =0.107, 0.0852 and 0.0746, respectively, which are back-propagated from the same complex amplitude distribution by using the exact diffraction kernel (or the quadratic-phase exponential kernel for the Fresnel approximation). Then the NRMS errors for the reconstructed objects in Figs. 3(a), 3(c) and 3(e) [or 3(b), 3(d) and 3(f)] are 0.393, 0.467 and 0.518 (or 0.583, 0.540 and 0.542), respectively. From these results, it is found that the NA valid for the Fresnel approximation in this 
measurement system is about 0.0746 or less because the appearances of the reconstructed objects for two different kernels become to resemble each other in Figs. 3(e) and 3(f).

We next consider the diffraction-limited depth resolution for the object reconstruction in the present system. As the criterion of the depth resolution, the depth limit is usually defined [23] by $\delta_{d}=\lambda / 2(\mathrm{NA})^{2}$. In the present system with $\mathrm{NA}=0.214$, the depth limit $\delta_{d}$ becomes to be about $0.218 \mu \mathrm{m}$, which corresponds to about eleven times the wavelength of the illuminating beam. Thus we investigated the reconstruction of an object containing a depth structure that is thicker than the depth limit $\delta_{d}$. Figures 4 to 7 show the results in the present system. The object has two stops consisting of plus and minus symbols, which are placed at distances of $1.6 \mu \mathrm{m}$ and $0.64 \mu \mathrm{m}$, respectively, in front of the object plane. Figure 4(a) shows the modulus of the complex amplitude at the plane immediately behind the plus symbol illuminated by a Gaussian beam, and Fig. 4(b) shows the modulus of the complex amplitude at the plane immediately behind the minus symbol placed at a distance of $0.96 \mu \mathrm{m}$ downstream from the plus symbol. Figures 4(c) and 4(d) show the modulus and phase of the complex amplitude, respectively, of the wavefront reached at the object plane. In Fig. 4(e), the noiseless modulus data in the detector plane is shown on the same gray scale as in Fig. 3(c), where the same array filter and parameters as in the first simulation were used. In order to compare reconstructed objects with ideal ones, we calculated the complex amplitude distributions back-propagated from the original complex amplitude distribution in the object plane. Figures 5(a) and 5(b) [or 5(c) and 5(d)] are the modulus and the phase of the ideal amplitude distribution, respectively, at distances of $0.64 \mu \mathrm{m}$ [or $1.6 \mu \mathrm{m}$ ] in front of the object plane, which is back-propagated from the complex amplitude consisting of the modulus and the phase in Figs. 4(c) and 4(d), respectively. It can be seen that there appears the influence of the minus symbol on the modulus 
in Figs. 5(c) and 5(d) when back-propagating from the complex amplitude in the object plane. Figure 6 (or 7) shows the reconstructed objects at locations some distances from the object plane in the noiseless (or noisy) case by using the same procedure as in the first simulation except for the use of four value of $\tau$ at the same spacing $0.0445 \mu \mathrm{m}$, which yielded the best reconstruction for the object's extent. The SNR in Fig. 7 becomes 129. The complex amplitude distribution at any position can be generated by the back-propagation of the reconstructed amplitude distribution in the object plane by using the convolution with the exact diffraction kernel in Eq. (7). Figures 6(a) and 6(b), 6(c) and 6(d), and 6(e) and 6(f) [or 7(a) and 7(b), 7(c) and 7(d), and 7(e) and 7(f)] show the moduli and phases of the back-propagated amplitude distributions in the noiseless (or noisy) case, which minimize the NRMS errors in comparing with the ideal complex amplitudes shown in Figs. 4(c) and 4(d), 5(a) and 5(b), and 5(c) and 5(d), respectively. In Figs. 6(a) and 6(b), 6(c) and 6(d), and 6(e) and 6(f) [or 7(a) and 7(b), 7(c) and 7(d), and 7(e) and 7(f)], the NRMS errors are $0.286,0.289$, and 0.289 (or $0.464,0.465$, and 0.466 ), respectively, and the focal positions of the reconstructed objects are $-0.32 \mu \mathrm{m}, 0.32 \mu \mathrm{m}$, and $1.28 \mu \mathrm{m}$ (or $-0.16 \mu \mathrm{m}, 0.48 \mu \mathrm{m}$, and $1.44 \mu \mathrm{m})$ from the object plane, respectively. From these results, it is found that the relative position of the two stops (plus and minus symbols) is retrieved from a single measurement data in the detector plane by using the present method. However, the absolute positions of the reconstructed objects have an error of $0.32 \mu \mathrm{m}$ in the noiseless case (or $0.16 \mu \mathrm{m}$ in the noisy case), and then the error of the absolute positions in the noiseless case is somewhat larger than the depth limit $\delta_{d}=0.218 \mu \mathrm{m}$. This is because the accuracy of the phase retrieval calculation is limited by systematic errors in the data such as errors in interpolation and aliasing in discrete Fourier transforms. 
The NRMS errors in the first simulation are larger than those in the second simulation. The reason is that such an object consisting of pulse-like functions, which contains many highfrequency components, is susceptible to the influence of aliasing in discrete Fourier transforms. On the other hand, it is inevitable that the phase retrieval from a high-NA intensity distribution becomes to be more sensitive to noises than that from a low-NA (i.e., Fresnel diffraction) intensity distribution, because the values of the high frequency components, which are contained in the high-NA intensity distribution, are generally smaller than those of the low frequency components.

\section{Conclusions}

We have considered the phase retrieval for the high-NA measurement system in applications to microscopy. In such high-NA systems, the Fresnel diffraction approximation, which is premised on almost all non-interferometric phase retrieval methods so far, cannot be utilized. Thus we have presented the extension of the phase retrieval method using an aperture-array filter to the object reconstruction based on the exact diffraction formula, which is suitable for the high-NA measurement system. In this extended method, the complex amplitude distribution in the diffraction plane is retrieved by regarding the measured intensity as the intensity of the Fresnel diffraction from a blurred object function, and then a high-resolved object function is reconstructed by back-propagating the retrieved complex amplitude distribution with the exact diffraction formula. It has been shown in computer simulations that the resolution of the reconstructed objects can be greatly improved by using the extended 
method. In addition, we have demonstrated that the object's structure in the depth direction can be reconstructed from a single measurement intensity distribution with accuracy of the diffraction-limited depth resolution, like holography. Consequently, there is a possibility that the present phase retrieval method provides a new technique of reconstructing the threedimensional information of an object from only a single measurement of a high-NA diffraction intensity distribution in a wide class of wave fields such as optical, $\mathrm{x}$-ray, electron, and atomic waves.

\section{Acknowledgements}

This research was supported by a Grant-in-Aid for Scientific Research from Japan Society for the Promotion of Science. 


\section{References}

1. H. Yamazaki, Y. Kohmura, T. Sakurai, and T. Ishikawa, "Reconstruction of complex-valued electron density with x-ray in-line holograms,” J. Opt. Soc. Am. A 23, 3171-3176 (2006).

2. A. Morlens, J. Gautier, G. Rey, P. Zeitoun, J. Caumes, M. Kos-Rosset, H. Merdji, S. Kazamias, K. Cassou, and M. Fajardo, "Submicrometer digital in-line holographic microscopy at 32 nm with high-order harmonics,” Opt. Lett. 31, 3095-3097 (2006).

3. A. Rosenhahn, R. Barth, F. Staier, T. Simpson, S. Mittler, S. Eisebitt, and M. Grunze, “Digital in-line soft x-ray holography with element contrast,” J. Opt. Soc. Am. A 25, 416-422 (2008).

4. S. Eisebitt, J. Lüning, W. F. Schlotter, M. Lörgen, O. Hellwig, W. Eberhardt, and J. Stöhr , "Lensless imaging of magnetic nanostructures by X-ray spectro-holography,” Nature 432, 885-888 (2004).

5. S. G. Podorov, K. M. Pavlov, and D. M. Paganin, “A non-iterative reconstruction method for direct and unambiguous coherent diffractive imaging,” Opt. Express 15, 9954-9962 (2007).

6. M. Guizar-Sicairos and J. R. Fienup, "Holography with extended reference by autocorrelation linear differential operation,” Opt. Express 15, 17592-17612 (2007).

7. S. Marchesini, S. Boutet, A. E. Sakdinawat, M. J. Bogan, S. Bajt, A. Barty, H. N. Chapman, M. Frank, S. P. Hau-riege, A. Szöke, C. Cui, D. A. Shapiro, M. R. Howells, J. C. H. Spence, J. W. Shaevitz, J. Y. Lee, J. Hajdu, and M. M. Seibert, “Massively parallel X-ray holography,” Nat. Photonics 2, 560-563 (2008).

8. J. R. Fienup, “Phase retrieval algorithms: a comparison,” Appl. Opt. 21, 2758-2769 (1982). 
9. M. Guizar-Sicairos and J. R. Fienup, "Phase retrieval with Fourier-weighted projections,” J. Opt. Soc. Am. A 25, 701-709 (2008).

10. H. M. L. Faulkner and J. M. Rodenburg, “Movable aperture lensless transmission microscopy: a novel phase retrieval algorithm,” Phys. Rev. Lett. 93, 023903 (2004).

11. M. R. Teague, “Deterministic phase retrieval: a Green's function solution,” J. Opt. Soc. Am. 73, 1434-1441 (1983).

12. T. E. Gureyev, A. Roberts, and K. A. Nugent, "Phase retrieval with the transport-of-intensity equation: matrix solution with use of Zernike polynomials,” J. Opt. Soc. Am. A 12, 19321941 (1995).

13. K. A. Nugent, D. Paganin and T. E. Gureyev, “A phase odyssey,” in Physics Today 54, 2732 (2001).

14. J. Miao, P. Charalambous, J. Kirz, and D. Sayre, “Extending the methodology of X-ray crystallography to allow imaging of micrometre-sized non-crystalline speciments,” Nature 400, 342-344 (1999).

15. H. N. Chapman, A. Barty, S. Marchesini, A. Noy, S. P. Hau-Riege, C. Cui, M. R. Howells, R. Rosen, H. He, J. C. H. Spence, U. Weierstall, T. Beetz, C. Jacobsen, and D. Shapiro, “Highresolution $a b$ initio three-dimensional x-ray diffraction microscopy,” J. Opt. Soc. Am. A 23, 1179-1200 (2006).

16. J. M. Rodenburg, A. C. Hurst, A. G. Cullis, B. R. Dobson, F. Pfeiffer, O. Bunk, C. David, K. Jefimovs, and I. Johnson, “Hard x-ray lensless imaging of extended objects,” Phys. Rev. Lett. 98, 034801 (2007). 
17. Y. Nishino, Y. Takahashi, N. Imamoto, T. Ishikawa, and K. Maeshima, ”Three-dimensional visualization of a human chromosome using coherent x-ray diffraction,” Phys. Rev. Lett. 102, 018101 (2009).

18. B. E. Allman, P. J. McMahon, J. B. Tiller, K. A. Nugent, D. Paganin, A. Barty, I. MacNulty, S. P. Frigo, Y. Wang, and C. C. Retsch, "Noninterferometric quantitative phase imaging with soft x rays,” J. Opt. Soc. Am. A 17, 1732-1743 (2000).

19. T. E. Gureyev, S. Nayo, S. W. Wilkins, D. Paganin, and A. W. Stevenson, "Quantitative inline phase-contrast imaging with multienergy x rays,” Phys. Rev. Lett. 86, 5827-5830 (2001).

20. N. Nakajima, "Noniterative phase retrieval from a single diffraction intensity pattern by use of an aperture array,” Phys. Rev. Lett. 98, 223901 (2007).

21. N. Nakajima, "Lensless coherent imaging by a deterministic phase retrieval method with an aperture-array filter,” J. Opt. Soc. Am. A 25, 742-750 (2008).

22. J. W. Goodman, Introduction to Fourier Optics, 2nd ed. (McGraw-Hill, New York, 1996).

23. J. Garcia-Sucerquia, W. Xu, S. K. Jericho, P. Klages, M. H. Jericho, and H. J. Kreuzer, “Digital in-line holographic microscopy,” Appl. Opt. 45 836-850 (2006).

24. N. Delen and B. Hooker, “Free-space beam propagation between arbitrarily oriented planes based on full diffraction theory: a fast Fourier transform approach,” J. Opt. Soc. Am. A 15, 857-867 (1998).

25. N. Nakajima, Phase retrieval using the properties of entire functions, in: P. W. Hawkes (Ed.), Advances in Imaging and Electron Physics, vol. 93, Academic, New York, 1995.

26. N. Nakajima, “Improvement of resolution for phase retrieval by use of a scanning slit,” Appl. Opt. 45, 5976-5983 (2006). 


\section{Figure Captions}

Fig. 1. Schematic diagram of the measurement system for the object reconstruction by the phaseretrieval method. The object function is reconstructed from a single intensity distribution of a diffracted wave through an array of square apertures.

Fig. 2. Object reconstruction from a high-numerical-aperture (NA) diffraction intensity ( NA $=0.214$ ): (a) modulus of an original object consisting of seven Gaussian functions. (b) and (d) are the moduli of reconstructed objects from noiseless and noisy, respectively, moduli of the intensity in the detector plane. (c) is the noiseless modulus in the detector plane. (e) is the modulus of another reconstructed object that is back-propagated from the same amplitude distribution as used in (b) by using the Fresnel integral instead of the first Rayleigh-Sommerfeld integral. The only picture of (c) is represented on a base-10 logarithmic grey scale of a normalized modulus truncated to $10^{-4}$ for display purposes.

Fig. 3. Examples of reconstructed objects by back-propagating with the diffraction kernels for spherical and parabolic waves in varying NA: (a), (c), and (e) [or (b), (d), and (f)] are the moduli of reconstructed objects from the retrieved complex amplitude distribution in the diffraction plane for $\mathrm{NA}=0.107,0.0852$, and 0.0746 , respectively, by use of the backpropagation with the spherical (or parabolic) diffraction kernel.

Fig. 4. Original object function containing a depth structure: The object consists of two stops of plus and minus symbols, which are placed at distances of $1.6 \mu \mathrm{m}$ and $0.64 \mu \mathrm{m}$, respectively, in front of the object plane. (a) and (b) are the moduli of the complex amplitudes in the planes immediately behind the plus and the minus stops, respectively, when illuminating with a Gaussian beam. (c) and (d) are the modulus and phase, 
respectively, of the complex amplitude in the object plane. (e) is the noiseless modulus in the detector plane, which is represented on the same gray scales as in Fig. 2(c).

Fig. 5. Ideal complex amplitudes generated by the back-propagation of the original complex amplitude in Figs. 4(c) and 4(d). (a) and (b) [or (c) and (d)] are the modulus and phase of the back-propagated complex amplitude at the position of $0.64 \mu \mathrm{m}$ (or $1.6 \mu \mathrm{m}$ ) in front of the object plane.

Fig. 6. Reconstruction of the object in Fig. 4 from noiseless modulus data: (a), (c), and (e) [or (b), (d), and (f)] are the moduli (or phases) of reconstructed objects at the focal positions of $-0.32 \mu \mathrm{m}, 0.32 \mu \mathrm{m}$, and $1.28 \mu \mathrm{m}$ from the object plane, respectively. The complex amplitudes at three positions minimize the NRMS errors in comparing with the corresponding ideal ones in Figs. 4 and 5.

Fig. 7. Reconstruction of the object in Fig. 4 from noisy modulus data: (a), (c), and (e) [or (b), (d), and (f)] are the moduli (or phases) of reconstructed objects at the focal positions of $-0.16 \mu \mathrm{m}, 0.48 \mu \mathrm{m}$, and $1.44 \mu \mathrm{m}$ from the object plane, respectively. The complex amplitudes at three positions minimize the NRMS errors in comparing with the corresponding ideal ones in Figs. 4 and 5. 


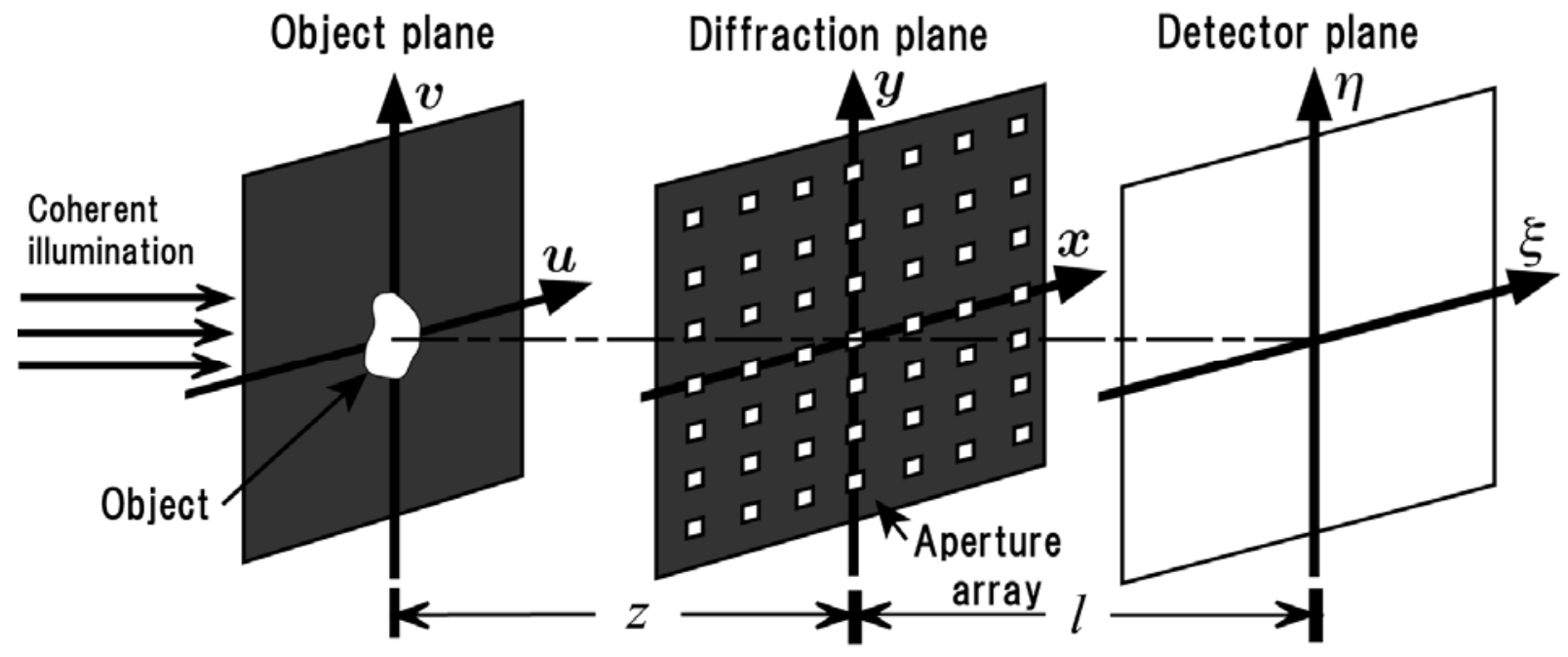

Fig. 1 


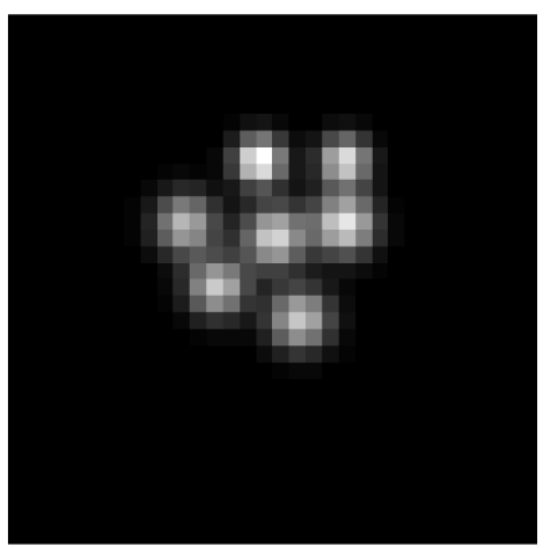

(a)

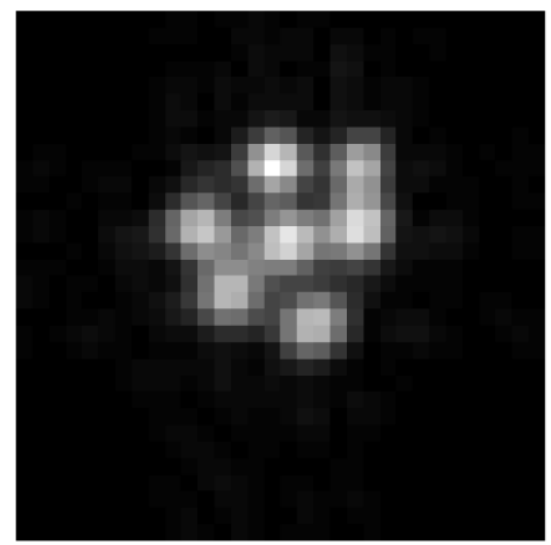

(b)

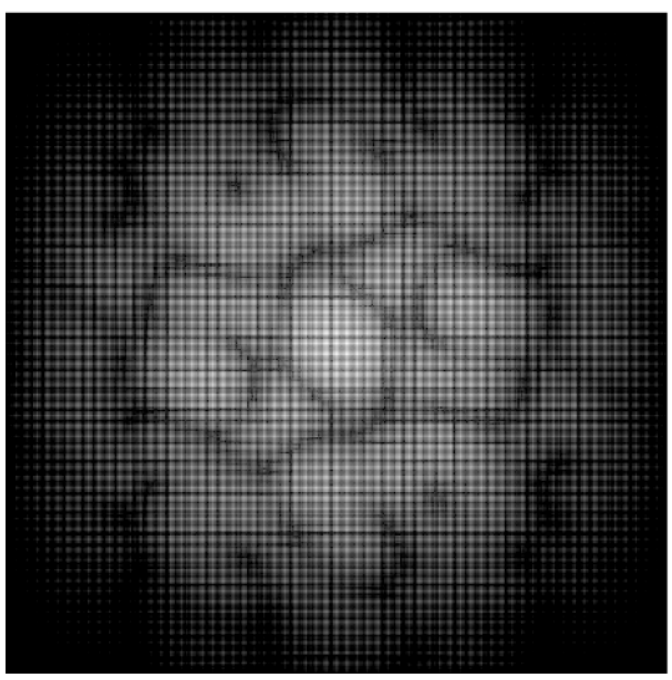

(c)

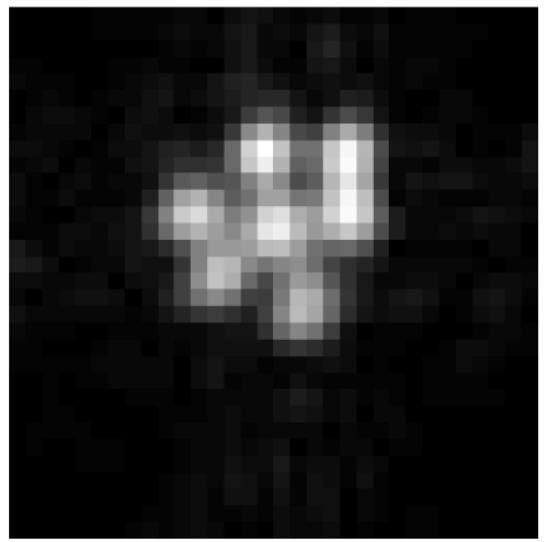

(d)

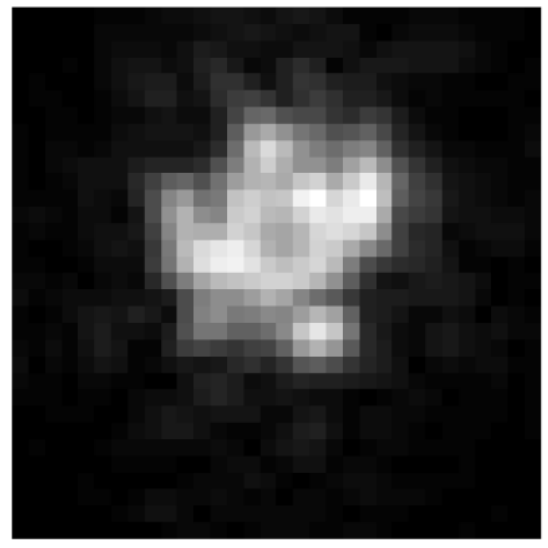

(e)

Fig. 2 


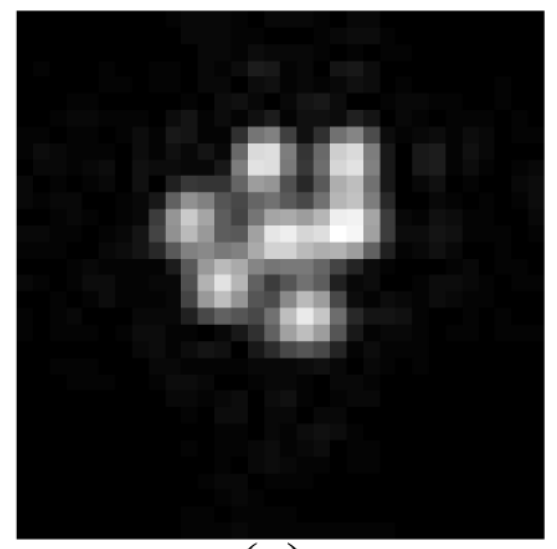

(a)

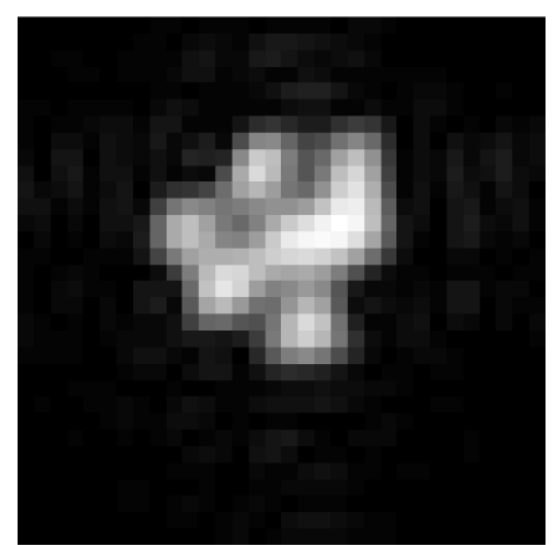

(c)

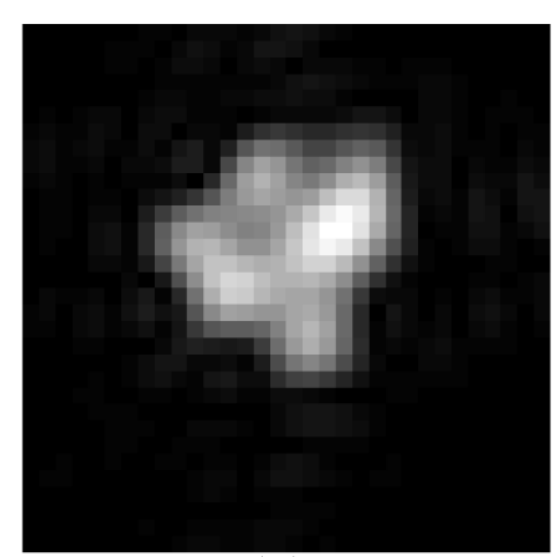

(e)

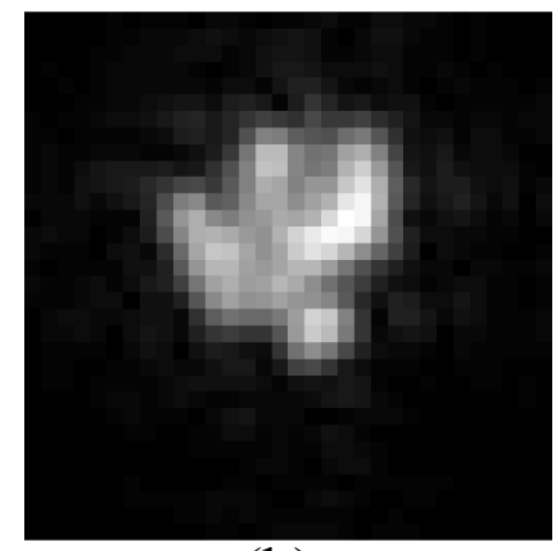

(b)

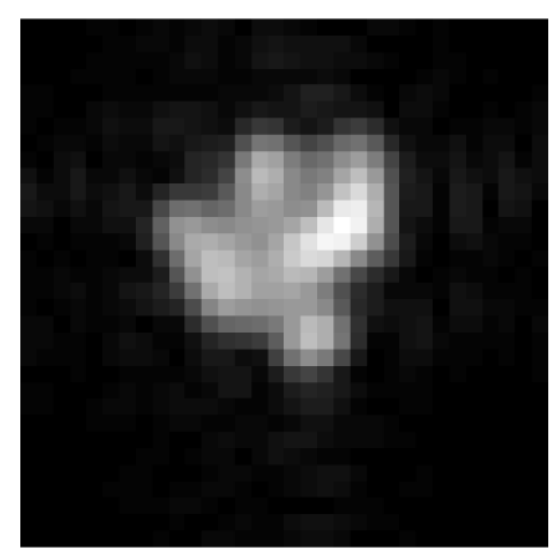

(d)

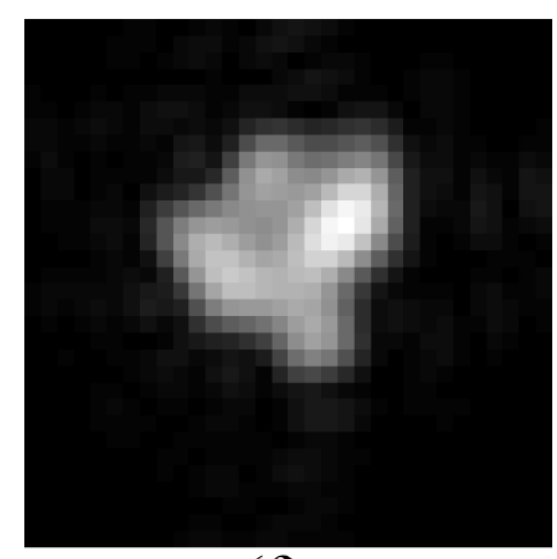

(f)

Fig. 3 


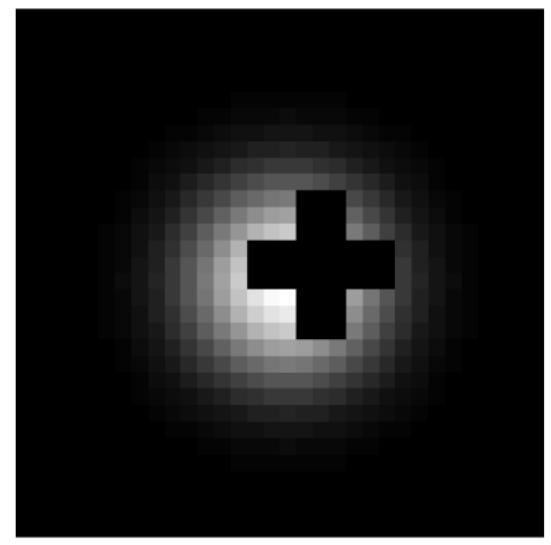

(a)

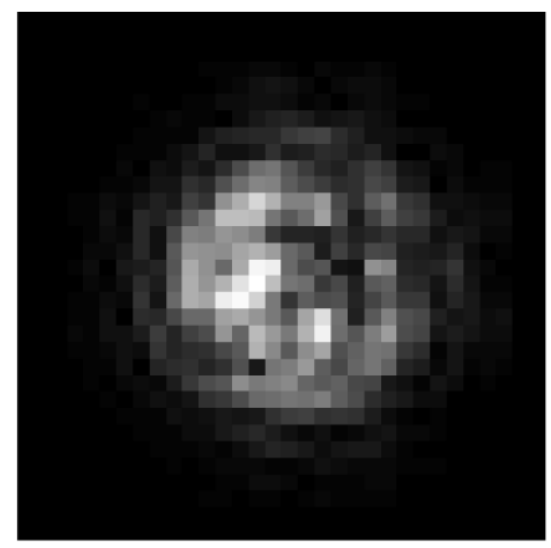

(c)

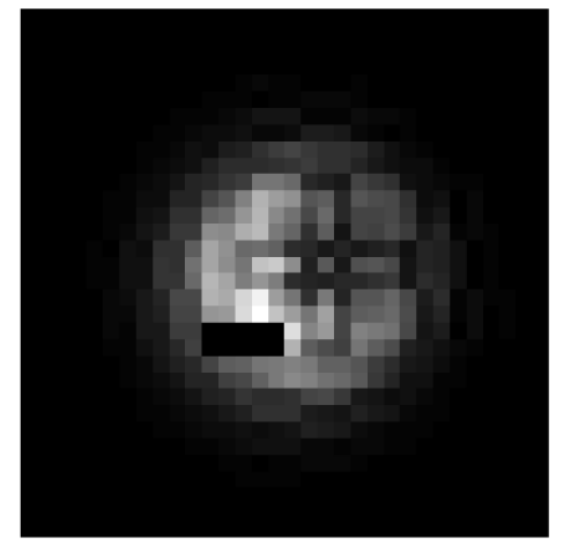

(b)

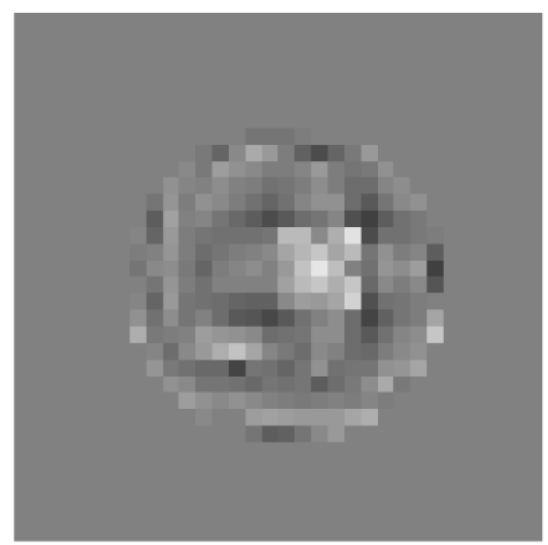

(d)

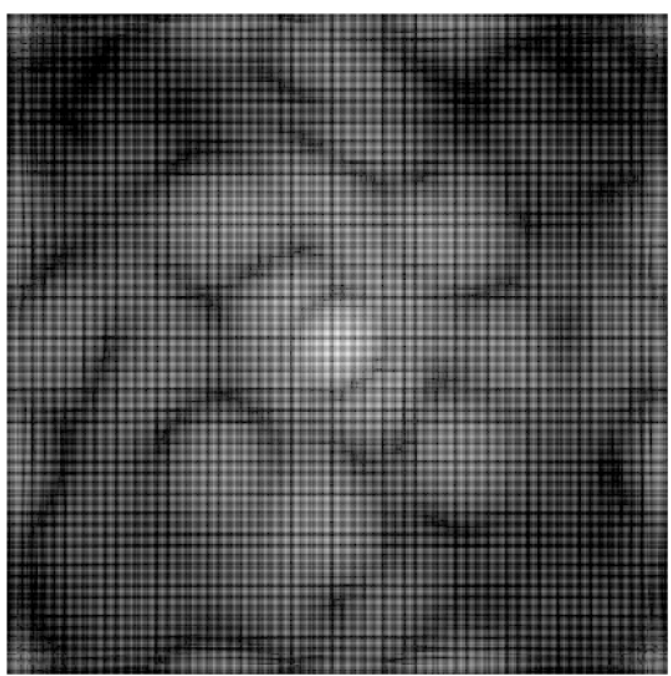

(e)

Fig. 4 


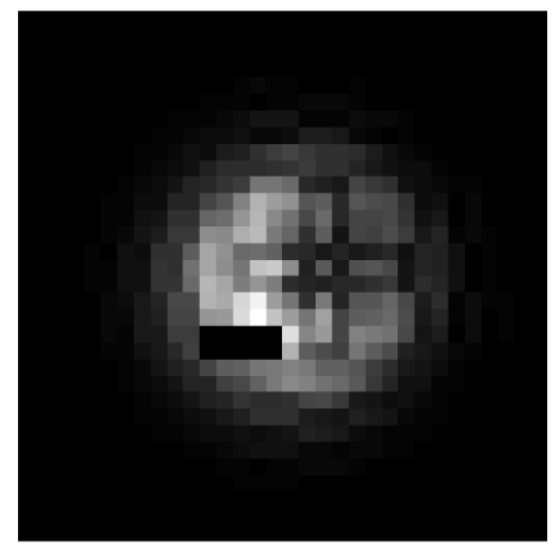

(a)

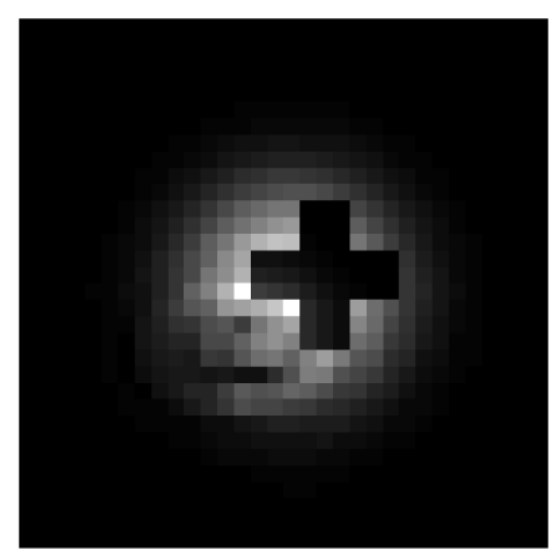

(c)

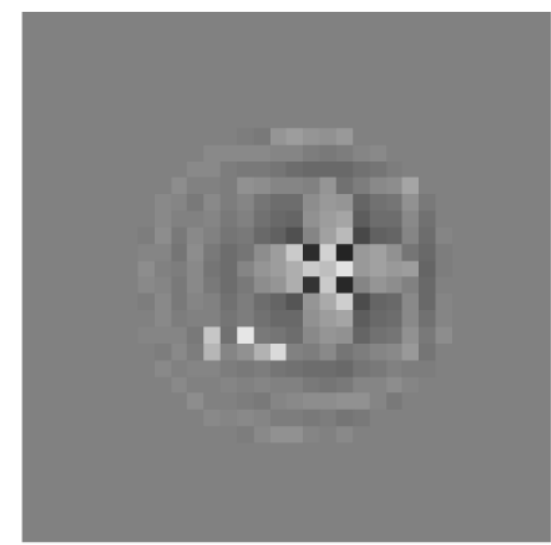

(b)

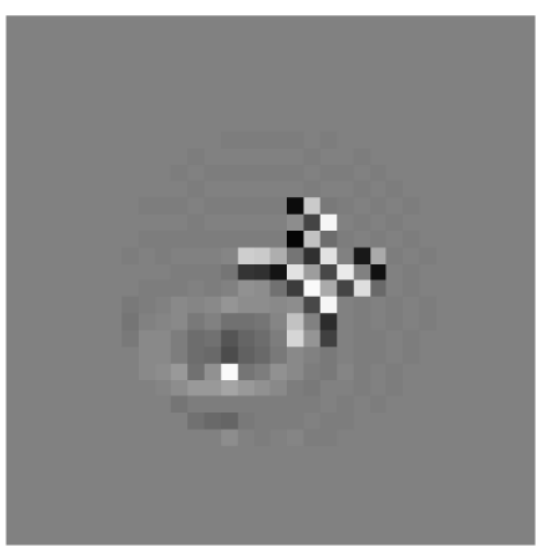

(d)

Fig. 5 


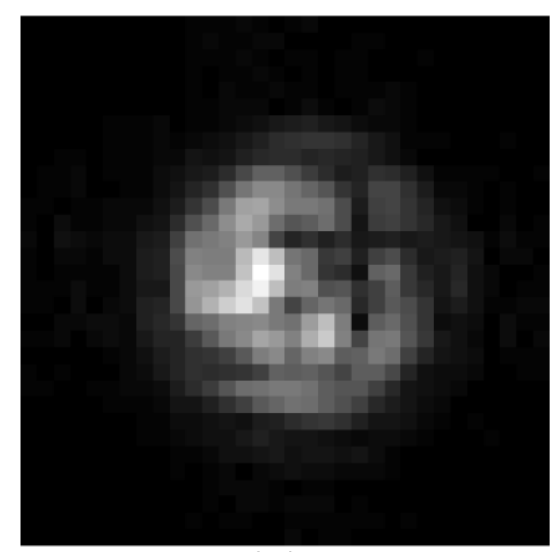

(a)

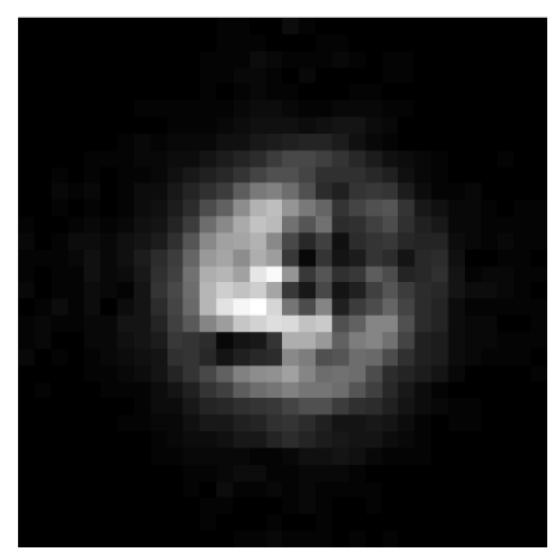

(c)

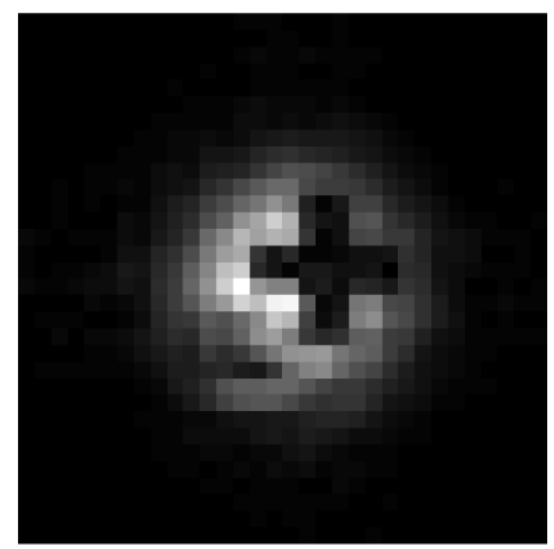

(e)

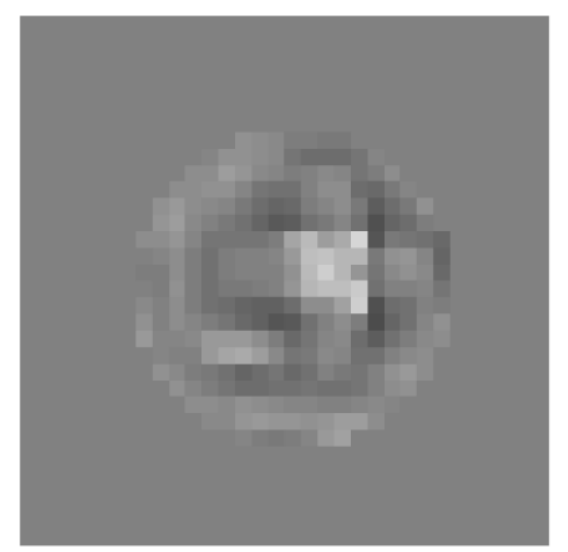

(b)

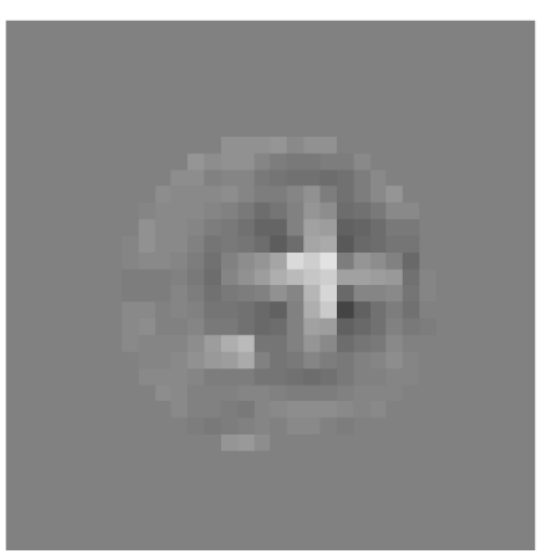

(d)

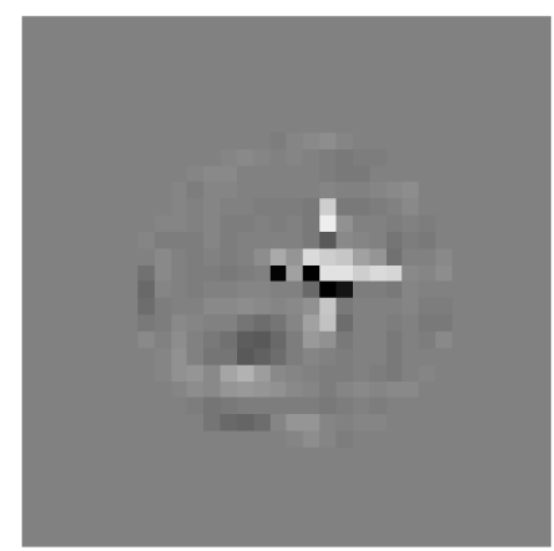

(f)

Fig. 6 


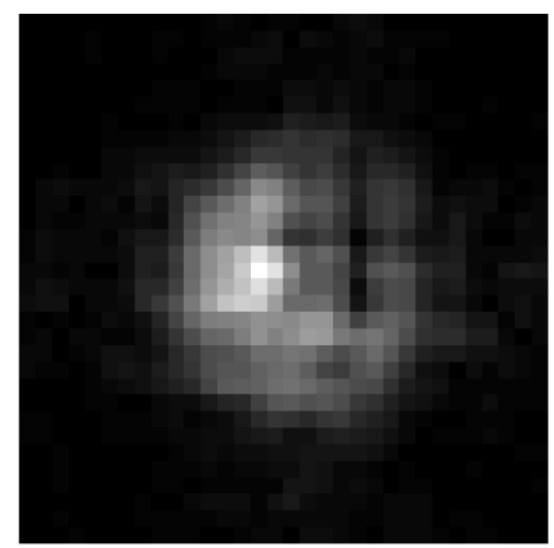

(a)

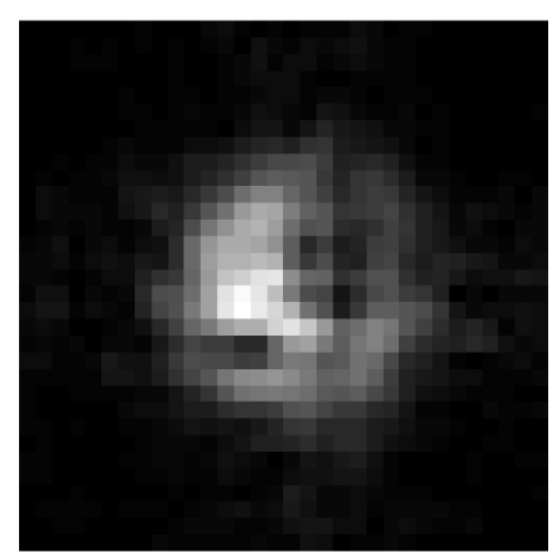

(c)

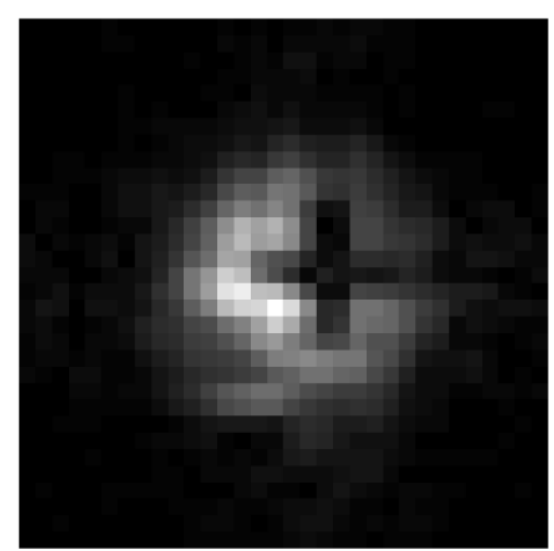

(e)

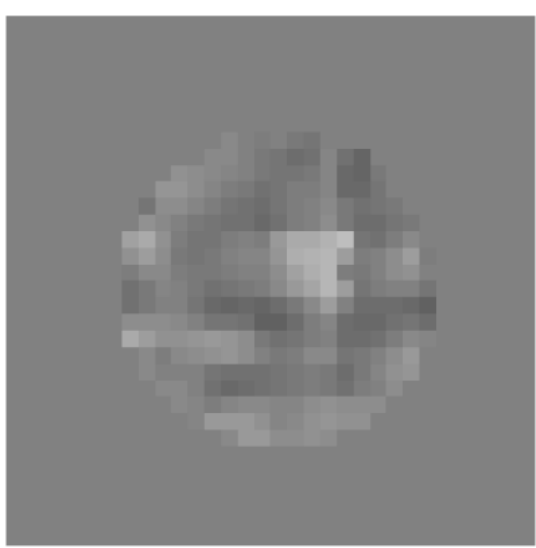

(b)

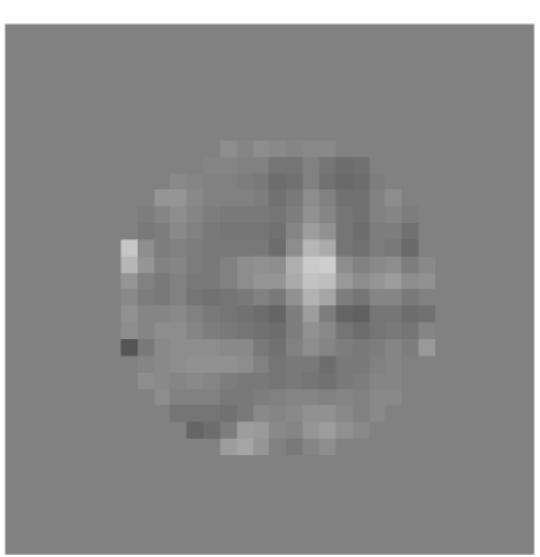

(d)

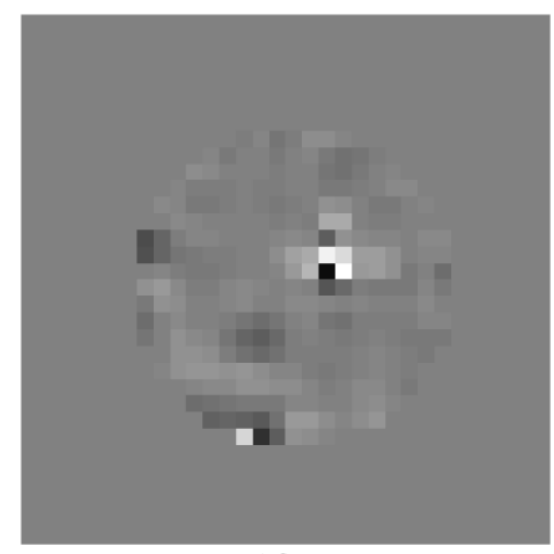

(f) 